\title{
COMPUTING SOME CONNECTIVITY INDICES OF $V$-PHENYLENIC NANOTUBES AND NANOTORI
}

\author{
Mohammad Reza Farahani \\ Department of Applied Mathematics, Iran University of Science and Technology \\ (IUST), Narmak, Tehran 16844, Iran
}

\begin{abstract}
Let $G$ be a simple connected graph in chemical graph theory and $e=u v$ be an edge of $G$. The Randić index $\chi(G)$ and sum-connectivity $X(G)$ of a nontrivial connected graph $G$ are defined as the sum of the weights $\frac{1}{\sqrt{d_{u} d_{v}}}$ and $\frac{1}{\sqrt{d_{u}+d_{v}}}$ over all edges $e=u v$ of $G$, respectively. In this paper, we compute Randić $\chi(G)$ and sum-connectivity $X(G)$ indices of $V$-phenylenic nanotubes and nanotori.

Keywords: molecular graph, $V$-phenylenic nanotubes, $V$-phenylenic nanotori, Randić connectivity index, sum-connectivity index.
\end{abstract}

\footnotetext{
${ }^{*}$ Corresponding author.

E-mail address: mr_farahani@mathdep.iust.ac.ir; mrfarahani88@gmail.com
}

(Mohammad Reza Farahani).

Copyright (c) 2015 Scientific Advances Publishers

2010 Mathematics Subject Classification: 05C05, 05C12.

Submitted by Jianqiang Gao.

Received September 23, 2015; Revised October 10, 2015 


\section{Introduction}

Let $G$ be a simple connected graph in chemical graph theory. The vertex set and edge set of $G$ denoted by $V(G)$ and $E(G)$, respectively, and its vertices correspond to the atoms and the edges correspond to the bonds [1-4].

There are many different kinds of topological indices or chemical indices. A chemical topological index is a numeric quantity from the structural graph of a molecule and is invariant on the automorphism of the graph. Some of them are distance based indices like Wiener index, some are degree based indices like the Randić index. The Randić index $\chi(G)$ of a graph $G$ is defined as

$$
\chi(G)=\sum_{e=u v \in E(G)} \frac{1}{\sqrt{d_{u} d_{v}}} .
$$

It is also known as connectivity index or branching index. Randić in 1975 [5] proposed this index for measuring the extent of branching of the carbon-atom skeleton of saturated hydrocarbons. There is also a good correlation between the Randic index and several physicochemical properties of alkanes: boiling points, surface areas, energy levels, etc.

In 2008, Zhou and Trinajstić introduced the sum-connectivity index $X(G)$ as

$$
X(G)=\sum_{v_{u} v_{v}} \frac{1}{\sqrt{d_{u}+d_{v}}},
$$

where $d_{u}$ and $d_{v}$ are the degrees of the vertices $u$ and $v$, respectively.

For a comprehensive survey of its mathematical properties, see the book of $\mathrm{Li}$ and Gutman [6], or recent survey of $\mathrm{Li}$ and Shi [7]. See also the books of Kier and Hall [8, 9] for chemical properties of this index.

In this paper, we compute these connectivity topological indices of $V$-phenylenic nanotubes and nanotori. 


\section{Main Results and Discussion}

In this section, Randić connectivity index $\chi(G)=\sum_{e=u v \in E(G)} \frac{1}{\sqrt{d_{u} d_{v}}}$ and sum-connectivity index $X(G)=\sum_{v_{u} v_{v}} \frac{1}{\sqrt{d_{u}+d_{v}}}$ of $V$-phenylenic nanotubes and nanotori are computed. Before present the main results, we recall some useful definitions and notations.

Definition 1 ([10]). Consider an arbitrary vertex $v$ with degree $d_{v}$ of simple connected graph $G=(V(G) ; E(G))$ and we denoted the minimum degree with $\delta=\operatorname{Min}\left\{d_{v} \mid v \in V(G)\right\}$ and the maximum degree with $\Delta=\operatorname{Max}\left\{d_{v} \mid v \in V(G)\right\}$. By according to the vertices degree, we have several partitions of vertex set $V(G)$ and edge set $E(G)$ of graph $G$, as follow:

$$
\begin{gathered}
\forall k: \delta \leq k \leq \Delta, \quad V_{k}=\left\{v \in V(G) \mid d_{v}=k\right\}, \\
\forall i: 2 \delta \leq i \leq 2 \Delta, \quad E_{i}=\left\{e=u v \in E(G) \mid d_{u}+d_{v}=i\right\}, \\
\forall j: \delta^{2} \leq j \leq \Delta^{2}, \quad E_{j}^{*}=\left\{u v \in E(G) \mid d_{u} \times d_{v}=j\right\} .
\end{gathered}
$$

Before going to calculate favourite connectivity indices, we divide the vertex set $V(V P H X[m, n])$ and the edge set $E(V P H X[m, n])$ of $V$-phenylenic nanotube in the following partitions:

$$
\begin{gathered}
V_{3}=\left\{v \in V(\operatorname{VPHX}[m, n]) \mid d_{v}=3\right\}, \\
V_{2}=\left\{v \in V(V P H X[m, n]) \mid d_{v}=2\right\}, \\
E_{5}=E_{6}{ }^{*}=\left\{u v \in E(V P H X[m, n]) \mid d_{u}+d_{v}=5 \& d_{u} \times d_{v}=6\right\}, \\
E_{6}=E_{9}{ }^{*}=\left\{u v \in E(V P H X[m, n]) \mid d_{u}+d_{v}=6 \& d_{u} \times d_{v}=9\right\} .
\end{gathered}
$$

Molecular graphs $V$-phenylenic nanotubes and nanotorus are two families of nano-structures that their structure are consist of cycles with length four, six, and eight by different compound. 
Professor Diudea denotes the $V$-phenylenic nanotubes and $V$-phenylenic nanotorus by $G=\operatorname{VPHX}[m, n]$ and $H=V P H Y[m, n]$, respectively [11]. Also, the general representations of these two kind of nano-structures are shown in Figure 1 and Figure 2. Readers can see the paper series [12-20], for a review historical details and further bibliography of $V$-phenylenic nanotubes and $V$-phenylenic nanotorus.

Theorem 1. Consider $V$-phenylenic nanotubes $G=V P H X[m, n]$ for every $m, n \in \mathbb{N}-\{1\}$. Then

The Randic connectivity index of $G$ is equal to

$$
\chi(V P H X[m, n])=\left(3 n+\frac{2 \sqrt{6}-5}{3}\right) m \approx(3 n-0.03367) m .
$$

The sum-connectivity index of $G$ is equal to

$$
X(V P H X[m, n])=\left(\frac{9 \sqrt{6} n}{6}+\left(\frac{4 \sqrt{5}}{5}-\frac{5 \sqrt{6}}{6}\right)\right) m \approx(3.6742 n-0.2524) m .
$$

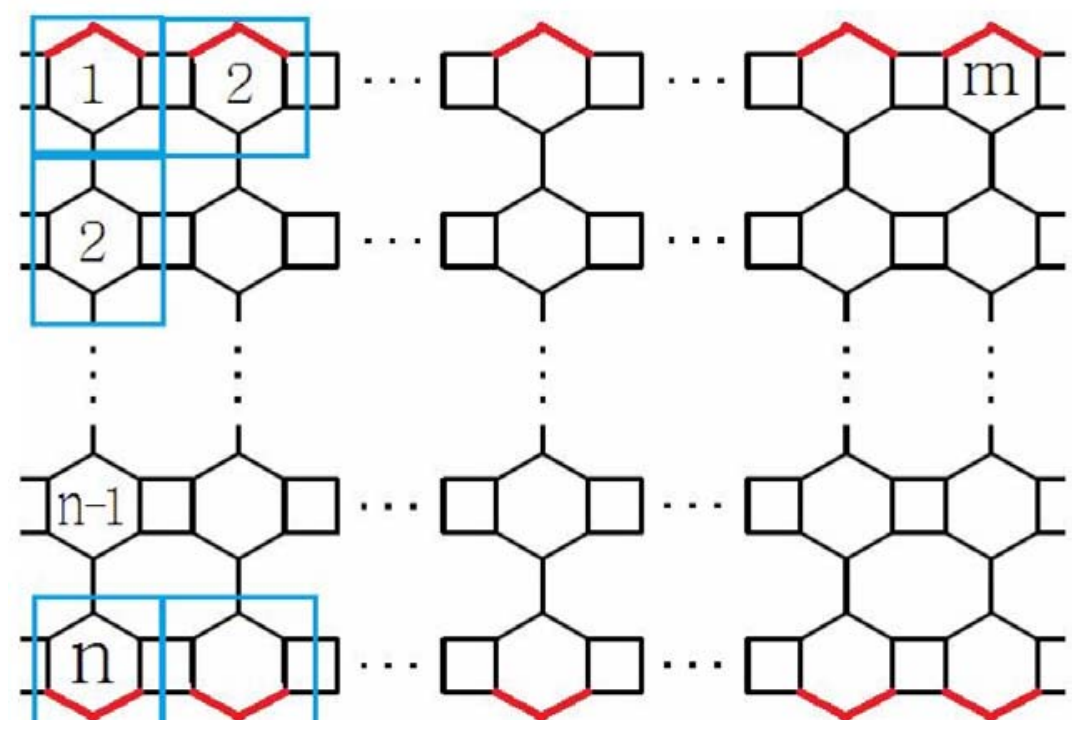

Figure 1. 2-D Lattice of the $V$-phenylenic nanotubes $G=V P H X[m, n]$. 
Proof of Theorem 1. Consider $V$-phenylenic nanotube $G=V P H X[m, n]$ $(m, n>1)$, since $G$ consist of several adjacent hexagon (or cycle $C_{6}$ ), we denote the number of repetition of these $C_{6}$ 's in the first row/column by $m$ and $n$, respectively.

Thus by look at the general case $V$-phenylenic nanotube $G=V P H X[m, n]$ in Figure 1, one can see that the number of vertices and edges in $G=\operatorname{VPHX}[m, n]$ are equal to $6 m n$ and $9 m n-m$, respectively. Since $\left|V_{2}\right|=m+m$ and $\left|V_{3}\right|=6 m n-2 m$. Then the number of edges of $G=\operatorname{VPHX}[m, n]$ will be $|E(V P H X[m, n])|=\frac{1}{2}[2(2 m)+3(6 m n-2 m)]$.

Now by according to Figure 2, we see that there exist the number of $2 m+2 m$ members in the edge partition $E_{5}$ or $E_{6}{ }^{*}$ (the red colour edges in Figure 1) and there are $9 m n-5 m$ members in the edge partition $E_{6}$ or $E_{9}{ }^{*}$ (the black colour edges in Figure 1).

Now, the Randić connectivity index, sum-connectivity index of $V$-phenylenic nanotube $G=\operatorname{VPHX}[m, n]$ are equal, respectively, as

$$
\begin{aligned}
\chi(V P H X[m, n]) & =\sum_{u v \in E(V P H X[m, n])} \frac{1}{\sqrt{d_{u} d_{v}}} \\
& =\sum_{e=u v \in E_{9}^{*}} \frac{1}{\sqrt{d_{u} d_{v}}}+\sum_{e=u v \in E_{6}^{*}} \frac{1}{\sqrt{d_{u} d_{v}}} \\
& =\frac{\left|E_{9}^{*}\right|}{\sqrt{9}}+\frac{\left|E_{6}^{*}\right|}{\sqrt{6}} \\
& =\frac{9 m n-5 m}{3}+\frac{4 m \sqrt{6}}{6} \\
& =\left(3 n+\frac{2 \sqrt{6}-5}{3}\right) m,
\end{aligned}
$$


and

$$
\begin{aligned}
X(V P H X[m, n]) & =\sum_{u v \in E(V P H X[m, n])} \frac{1}{\sqrt{d_{u}+d_{v}}} \\
& =\sum_{e=u v \in E_{6}} \frac{1}{\sqrt{d_{u}+d_{v}}}+\sum_{e=u v \in E_{5}} \frac{1}{\sqrt{d_{u}+d_{v}}} \\
& =\frac{\left|E_{6}\right|}{\sqrt{6}}+\frac{\left|E_{5}\right|}{\sqrt{5}} \\
& =\left(\frac{9 \sqrt{6} n}{6}+\left(\frac{4 \sqrt{5}}{5}-\frac{5 \sqrt{6}}{6}\right)\right) m .
\end{aligned}
$$

Theorem 2. Let $G$ be the $V$-phenylenic nanotorus $H=V P H Y[m, n]$, $\forall m, n>1$. Then

The Randic connectivity index of $H$ is equal to $\chi(V P H Y[m, n])=3 m n$.

The sum-connectivity index of $H$ is equal to $X(V P H Y[m, n])=\frac{3 \sqrt{6} m n}{2}$

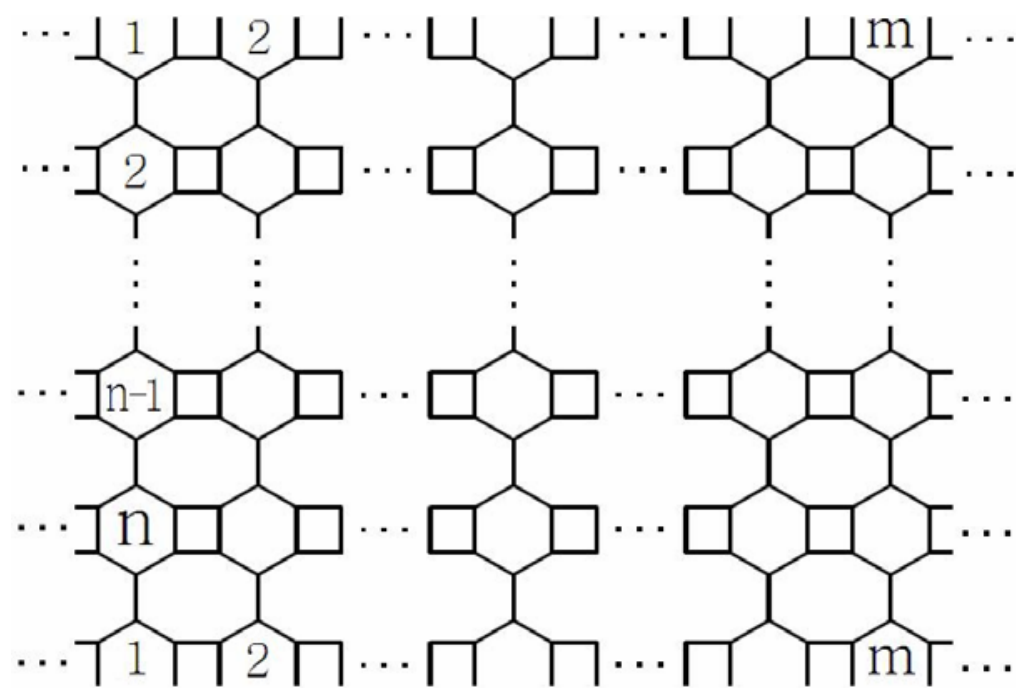

Figure 2. 2-D Lattice of the $V$-phenylenic nanotori $H=V P H Y[m, n]$. 
Proof of Theorem 2. Consider $V$-phenylenic nanotori $H=V P H Y[m, n]$ $(\forall m, n>1)$, where $m$ and $n$ be the number of hexagon in the first row and column in $H=V P H Y[m, n]$. From the structure of $V$-phenylenic nanotori in Figure 2, it is easy to see that $\operatorname{VPHY}[m, n]$ is a cubic graph (all its vertices have degree three).

Thus, the vertex partition $V_{3}$ is equal with $V(V P H Y[m, n])$ and $\left|V_{3}\right|=|V(\operatorname{VPHY}[m, n])|=6 m n$. Also, therefore the edge partitions $E_{6}$ or $E_{9}{ }^{*}$ are equal with $E(\operatorname{VPHY}[m, n])$ and $\left|E_{6}\right|=\left|E_{9}{ }^{*}\right|=9 m n$.

And these imply that

$$
\chi(V P H Y[m, n])=\sum_{u v \in E(V P H Y[m, n])} \frac{1}{\sqrt{d_{u} d_{v}}}=\frac{|E|}{\sqrt{9}}=3 m n,
$$

and

$$
X(V P H Y[m, n])=\sum_{u v \in E(V P H Y[m, n])} \frac{1}{\sqrt{d_{u}+d_{v}}}=\frac{|E|}{\sqrt{6}}=\frac{3 \sqrt{6} m n}{2} .
$$

Now, by computing Randić connectivity index $\chi(V P H Y[m, n])$, sumconnectivity index $X(\operatorname{VPHY}[m, n])$ for every $m, n \in \mathbb{N}-\{1\}$, the proof of Theorem 2 is completed.

\section{Conclusion}

In this paper, we focus on the connected structure of two kind of nanostructures " $V$-phenylenic nanotubes $G=V P H X[m, n]$ and $V$-phenylenic nanotorus $H=\operatorname{VPHY}[m, n]$ ”. Also, we count some connectivity indices "Randić connectivity index and sum-connectivity index" of them. 


\section{References}

[1] R. Todeschini and V. Consonni, Handbook of Molecular Descriptors, Wiley, Weinheim, 2000.

[2] N. Trinajstić, Chemical Graph Theory, CRC Press, Boca Raton, FL, 1992.

[3] N. Trinajstić and I. Gutman, Mathematical Chemistry, Croat. Chem. Acta 75 (2002), 329-356.

[4] I. Gutman and O. E. Polansky, Mathematical Concepts in Organic Chemistry, Springer-Verlag, New York, 1986.

[5] M. Randić, On characterization of molecular branching, J. Amer. Chem. Soc. 97 (1975), 6609-6615.

[6] X. Li and I. Gutman, Mathematical Aspects of Randić-Type Molecular Structure Descriptors, in: Mathematical Chemistry Monographs, Vol. 1, Kragujevac, 2006.

[7] L. B. Kier and L. H. Hall, Molecular Connectivity in Chemistry and Drug Research, Academic Press, New York, 1976.

[8] L. B. Kier and L. H. Hall, Molecular Connectivity in Structure-Activity Analysis, Research Studies Press, Wiley, Chichester, UK, 1986.

[9] X. Li and Y. Shi, A survey on the Randić index, MATCH Commun. Math. Comput. Chem. 59 (2008), 127-156.

[10] M. R. Farahani, Some connectivity indices and Zagreb index of polyhex nanotubes, Acta Chim. Slov. 59 (2012), 779-783.

[11] M. V. Diudea, Fuller. Nanotub. Carbon Nanostruct. 10 (2002), 273.

[12] V. Alamian, A. Bahrami and B. Edalatzadeh, $P I$ polynomial of $V$-phenylenic nanotubes and nanotori, Int. J. Mol. Sci. 9 (2008), 229-234.

[13] J. Asadpour, Some topological polynomial indices of nanostructures, Optoelectron. Adv. Mater.-Rapid Commun. 5(7) (2011), 769-772.

[14] A. Bahrami and J. Yazdani, Vertex $P I$ index of $V$-phenylenic nanotubes and nanotori, Digest Journal of Nanomaterials and Biostructures 4(1) (2009), 141-144.

[15] M. Davoudi Monfared, A. Bahrami and J. Yazdani, PI polynomial of $V$-phenylenic nanotubes, Digest Journal of Nanomaterials and Biostructures 5(2) (2010), 441-445.

[16] M. Ghorbani, H. Mesgarani and S. Shakeraneh, Computing GA index and ABC index of $V$-phenylenic nanotube, Optoelectron. Adv. Mater.-Rapid Commun. 5(3) (2011), 324-326.

[17] N. Prabhakara Rao and K. L. Lakshmi, Eccentricity connectivity index of $V$-phenylenic nanotubes, Digest Journal of Nanomaterials and Biostructures 6(1) (2010), 81-87. 
[18] M. R. Farahani, Computing $\mathrm{GA}_{5}$ index of $V$-phenylenic nanotubes and nanotori, Int. J. Chem Model 5(4) (2013), 479-484.

[19] M. R. Farahani, Computing fourth atom-bond connectivity index of $V$-phenylenic nanotubes and nanotori, Acta Chimica Slovenica 60(2) (2013), 429-432.

[20] M. R. Farahani, Computing theta polynomial and theta index of $V$-phenylenic planar, nanotubes and nanotoris, International Journal of Theoretical Chemistry 1(1) (2013), 01-09. 